\title{
Publisher's Note: Excitons in core-only, core-shell and core-crown CdSe nanoplatelets: Interplay between in-plane electron-hole correlation, spatial confinement, and dielectric confinement
}

[Phys. Rev. B 96, 035307 (2017)]

Fernando Rajadell, Juan I. Climente, and Josep Planelles

(Received 7 September 2017; published 12 September 2017)

DOI: 10.1103/PhysRevB.96.119904

This paper was published online on 24 July 2017 with typographical errors in Eqs. (9) and (12), and in the text on page 3. Equation (9) should read as

$$
N=\sqrt{\frac{2}{\pi}} \frac{8}{\left(L_{x} L_{y}\right)^{1 / 2} L_{z}}\left(\frac{1}{a^{2}}+\frac{a}{2} \sum_{i=x, y} \frac{1}{\left(a^{2}+k_{i}^{2}\right)^{3 / 2}}+\frac{a}{4} \frac{1}{\left(a^{2}+k_{x}^{2}+k_{y}^{2}\right)^{3 / 2}}\right)^{-1 / 2} .
$$

Equation (12) should read as

$$
S_{e h}^{2}=N^{2}\left(\frac{L_{x}}{2}\right)^{2}\left(\frac{L_{y}}{2}\right)^{2}\left(\frac{L_{z}}{2}\right)^{2} .
$$

On page 3, right-hand column, the second line should read as "element, and $S_{e h}^{2}=\left|\left\langle\Psi\left|\delta_{r_{e}, r_{h}}\right| 0\right\rangle\right|^{2}$ the electron-hole (envelope function) overlap." The paper has been corrected as of 8 September 2017. The equations and text are incorrect in the printed version of the journal. 